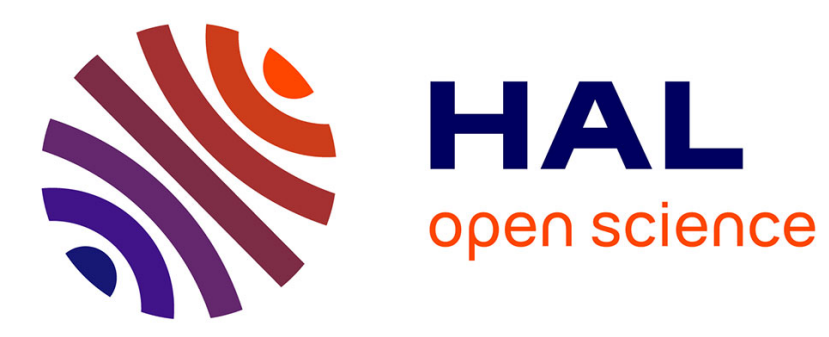

\title{
Twinned gold nanoparticles under growth: bipyramids shape controlled by environment
}

\author{
Z. Cansu Canbek, Robinson Cortes-Huerto, Fabienne Testard, Olivier Spalla, \\ Simona Moldovan, Ovidiu Ersen, Andreas Wisnet, Guillaume Wang, Jacek \\ Goniakowski, Claudine Noguera, et al.
}

\section{To cite this version:}

Z. Cansu Canbek, Robinson Cortes-Huerto, Fabienne Testard, Olivier Spalla, Simona Moldovan, et al.. Twinned gold nanoparticles under growth: bipyramids shape controlled by environment. Crystal Growth \& Design, 2015, 15 (8), pp.3637-3644. 10.1021/acs.cgd.5b00121 . hal-01187806

\section{HAL Id: hal-01187806 https://hal.science/hal-01187806}

Submitted on 7 Jul 2020

HAL is a multi-disciplinary open access archive for the deposit and dissemination of scientific research documents, whether they are published or not. The documents may come from teaching and research institutions in France or abroad, or from public or private research centers.
L'archive ouverte pluridisciplinaire HAL, est destinée au dépôt et à la diffusion de documents scientifiques de niveau recherche, publiés ou non, émanant des établissements d'enseignement et de recherche français ou étrangers, des laboratoires publics ou privés. 


\title{
Twinned Gold Nanoparticles under Growth: Bipyramids Shape Controlled by Environment
}

\author{
Z. Cansu Canbek, ${ }^{\dagger}$ Robinson Cortes-Huerto, ${ }^{\dagger, \$, \$}$ Fabienne Testard,,$^{* \dagger}$ Olivier Spalla, ${ }^{\dagger}$ Simona Moldovan," \\ Ovidiu Ersen," Andreas Wisnet, ${ }^{\#}$ Guillaume Wang, ${ }^{\perp}$ Jacek Goniakowski, ${ }^{\$, \$}$ Claudine Noguera, ${ }^{\$, \$}$ \\ and Nicolas Menguy
}

${ }^{\dagger}$ CEA Saclay, DSM/IRAMIS/NIMBE/LIONS, UMR CEA/CNRS 3685, 91191 Gif sur Yvette, France

${ }^{\ddagger}$ Université Pierre et Marie Curie, IMPMC, UMR 7590 CNRS, Campus Jussieu, 75252 Cedex 05 Paris, France

${ }^{\S}$ CNRS, INSP, UMR7588, F-75252 Cedex 05 Paris, France

${ }^{\$}$ UPMC Univ Paris 06, INSP, UMR7588, F-75252 Cedex 05 Paris, France

"Institut de Physique et Chimie des Matériaux de Strasbourg (IPCMS), UMR 7504, CNRS- Université de Strasbourg (UdS), 23 rue du Lœss, 67034 cedex 2 Strasbourg, France

${ }^{\perp}$ Université Paris Diderot-Paris7, Laboratoire Matériaux et Phénomènes Quantiques, Bâtiment Condorcet, 7021 F-75205 Cedex 13 Paris, France

\# Department of Chemistry and Center for NanoScience (CeNS), Ludwig Maximilians University 5LMU, Buternandstrasse 11,81377 Munich, Germany

\begin{abstract}
The structure of $(\mathrm{Au})$ bipyramids, very promising for their specific plasmonic properties, is fully analyzed for gradual stages of growth. From combined use of HRTEM (high resolution transmission electronic microscopy) in specific conditions and SAED (selected area electronic diffraction) analysis, the atomic structure of these elongated objects of different sizes is extracted (from 2 to $60 \mathrm{~nm}$ ). In the case of silver(I)assisted growth from citrate capped seeds, a clear pentatwinned structure, made by the superimposition of specific pairs of crystallographic zones is found for the different size nanoparticles representing the different steps of the growing bipyramids. The experimental results were analyzed in the framework of a recent atomistic approach developed for metal-environment interactions to account for the stability of multitwinned nanorods or bipyramids in a complex environment. Efficient in describing the

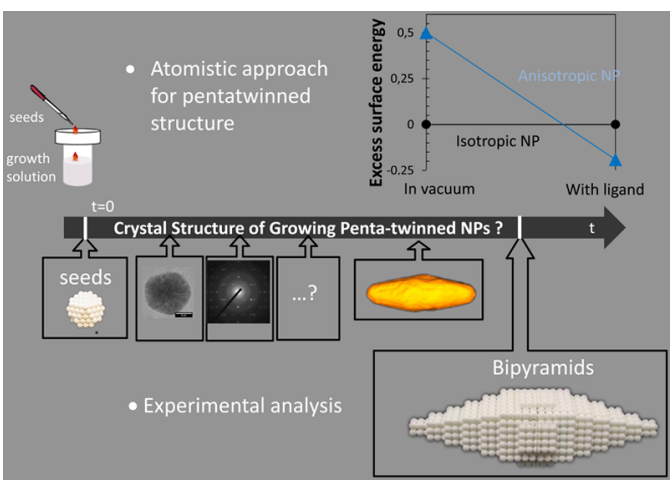
multitwinned nanoparticles and the drastic effects of environment on the

stabilization of elongated multitwinned shapes, this model supports the experimental features of bipyramids and nanorods formation from citrate seeds as a function of the nature of the surface chemical ligands: citrate seeds in a growth solution prepared without silver(I) yields the formation of a few pentatwinned nanorods, while the silver(I)-assisted growth process mainly leads to pentatwinned bipyramids. The conservation of a bulk pentatwinned structure inherited from isotropic decahedral (i-Dh) seeds all along the growth is retrieved by this approach for two different environments.
\end{abstract}

\section{INTRODUCTION}

The unique physical and chemical properties of anisotropic metallic nanoparticles (NPs) have inspired researchers to improve the control over their shape and size distribution. ${ }^{1,2}$ Today, beyond the several synthetic routes for anisotropic metallic NPs described in the literature, ${ }^{3-5}$ it is still a challenge to optimize the yield for a given anisotropic shape and to fully explain the mechanisms promoting the anisotropy (in particular when considering the materials crystallizing in a FCC phase).

In noble metal NPs, the particular case of gold $(\mathrm{Au})$ has drawn strong interest due to the plasmonic properties of $\mathrm{Au}$ NPs associated with their important potential applications in various fields (imaging, cancer therapy, electronic, detection, etc.). ${ }^{6}$ The most classical way to control the anisotropic shape of Au NPs is the seed mediated approach, which separates the nucleation and growth stages in time. ${ }^{2,7}$ This method involves the preparation of small precursors (seeds) in a first step, that grow into desired morphologies in a second step after their addition into a growth solution composed of complex chemical reagents. ${ }^{1,7,8}$ The seeded growth method has been widely utilized to synthesize multiple geometries including nanorods, cubes, plates, spherical particles, bipyramids, etc. ${ }^{4,9,10}$ with a main interest for nanorods. 
It is only recently that bipyramids (first considered as a byproduct) appeared to be very promising due to the high plasmon enhancement properties of their sharp tips. ${ }^{11}$ However, water synthetic processes are always polluted by other shapes with required postsynthetic purification. A better understanding of the reaction mechanism should help to improve the control over the final shapes.

Liu and Guyot-Sionnest ${ }^{12}$ were the first to identify the crucial importance of the seed structure in seeded growth methods. From transmission electron microscopy (TEM) images, they compare the seed structure to their associated final shapes after growth in silver(I)-CTAB growth solution. Two populations were identified; single crystal seeds (produced in CTAB) with a preferential growth into single crystal nanorods, and multitwinned seeds (produced in citrate solution), which grew preferentially into bipyramids. Even if the resolution of the TEM observations was not sufficient to clearly identify the precise seed crystal structure, these experiments pointed out the importance of defects in the seeds prior to their growth.

The nature of the growth solution also plays an important role. For instance, previously mentioned citrate seeds (multitwinned), utilized to obtained bipyramids with a growth solution containing silver(I), could also form pentatwinned nanorods (with an extremely low yield) in the absence of added silver in the growth solution. ${ }^{13}$ The anisotropic shape is clearly dictated by the complex mutual influence of the initial seed structure and the nature of the capping agent in the growth solution.

Concerning the precise role of the seeds, their polydispersity associated with their instability over time makes it difficult to define a clear roadmap to synthesize $100 \%$ of a desired anisotropic Au NP. The alternative seedless synthesis employed for the production of single crystalline gold nanorods also leads to a final state polluted by isotropic morphologies. ${ }^{14,15}$ The only way to identify the precise role played by the seeds in the formation of anisotropy is to determine the crystal structure of the nanoparticles during their formation, even though this presents a real challenge. In 2010, Carbo-Argibay et al. ${ }^{16}$ and Katz-Boon et al. ${ }^{17}$ took advantage of the recent development of electron tomography to elucidate the precise crystal structure of the final gold nanorods obtained from $C T A B$ capped seeds (single crystal). They evidenced the presence of high index facets on monocrystalline nanorods (obtained by the seed mediated method) in opposition to the structure of the pentatwinned nanorods (obtained by electrochemical method) described by Wang et al. in $1999 .{ }^{8}$ Recently, Zhou et al. ${ }^{18}$ utilized the high chemical and colloidal stability of large decahedral seeds $(>25$ $\mathrm{nm}$ ) to follow their growth into bipyramids $(D \approx 73 \mathrm{~nm}, L \approx 223$ $\mathrm{nm}$,) with good control over the shape and size. They have demonstrated that the bipyramids are derived from pentatwinned decahedra through a single step growth of the seeds. The use of such a strategy is, however, difficult to realize under classical conditions where the small size of seeds $(<3-5 \mathrm{~nm})$ limits their stability and characterization (particularly in CTAB water solution).

Furthermore, the structure of the bipyramids is still a matter of debate. Liu and Guyot-Sionnest ${ }^{12}$ were the first to analyze the final bipyramids by high resolution transmission electronic microscopy (HRTEM) and selected area electronic diffraction (SAED). From analogies with copper pentatwinned nanorods, ${ }^{5}$ they have stated that bipyramids $(D \approx 30 \mathrm{~nm}, L \approx 110 \mathrm{~nm}, \mathrm{AR} \approx$ 3.6) bear penta-fold twinned structures around their growth axis with probably high order external side facets. Such a penta-fold twinned model for bipyramids has been supported by various groups. ${ }^{12,19,20}$ Recently, Burgin and co-workers ${ }^{21}$ have investigated the structure of Au bipyramids $(D \approx 36 \mathrm{~nm}, L \approx 120 \mathrm{~nm}$, $\mathrm{AR} \approx 3.3$ ) by electron tomography and revealed an irregular 6 fold twinning structure with highly stepped, dominant $\{151\}$ side facets without any detail concerning the type of twinning. This surprising hexagonal section is also mentioned by Yoo et al. ${ }^{22}$ in their study on bipyramids obtained with silver(I)-assisted growth using cetyltrimethylammonium chloride (CTAC) and salicylic acid as surfactants. The versatility of the seeded growth synthesis and the difficulty in stabilizing the seed solution after their production (size $<3 \mathrm{~nm}$ ) can explain the different structures found for the bipyramids and their precursors.

The goal of this present work is to report a full characterization of $\mathrm{Au}$ bipyramids and their associated formation mechanism, starting from initial isotropic decahedra (i-Dh). Here, combining multiple techniques, we have revisited the crystal growth of bipyramidal Au nanostructures. We have investigated the growth mechanism from seeds to bipyramids, employing state of the art of TEM (HRTEM and electron tomography) combined with a theoretical approach (based on an atomistic approach for metal environment interaction) in order to model the effect of the environment on the stabilization of anisotropic multitwinned nanoparticles.

\section{EXPERIMENTAL SECTION}

2.1. Materials. Cetyltrimethylammonium bromide (CTAB, 99\%) was purchased from G-Biosciences; chloroauric acid (extra pure), silver nitrate (extra pure), sodium borohydride (98\%), trisodium citrate, Lascorbic acid (AA), and 5-bromosalycilic acid were obtained from Sigma. All these compounds were used without further purification. All glassware was cleaned with aqua regia and abundantly washed with distilled water before all the experiments.

2.2. Methods. 2.2.1. Chemical Synthesis of Au Bipyramids. 2.2.1.1. Synthesis of Seeds. Preparation of the so-called multitwinned seeds stabilized by citrate followed the pioneering work of Murphy's group. ${ }^{1}$ Typical synthesis involves the addition of $0.3 \mathrm{~mL}$ of ice cold 0.01 $\mathrm{M} \mathrm{NaBH}_{4}$ to a mixture of $10 \mathrm{~mL}$ of $0.25 \mathrm{mM} \mathrm{HAuCl}_{4}$ and $0.25 \mathrm{mM}$ sodium citrate under vigorous stirring at $28{ }^{\circ} \mathrm{C}$. The mixture color immediately turns to orange red. Citrate-stabilized seeds are aged for $3 \mathrm{~h}$ prior to use to ensure the full decomposition of excess borohydride in the solution.

2.2.1.2. Synthesis of Bipyramids. The chemical conditions for the reference synthesis are defined as follows: a growth solution is prepared by addition of $45 \mu \mathrm{L}$ of $10 \mathrm{mM} \mathrm{AgNO}_{3}$ and $100 \mu \mathrm{L}$ of $0.05 \mathrm{M} \mathrm{HAuCl}_{4}$ to a $10 \mathrm{~mL}$ solution of $0.1 \mathrm{M} \mathrm{CTAB}$, followed by the addition of $75 \mu \mathrm{L}$ of $0.1 \mathrm{M}$ ascorbic acid. Finally, $400 \mu \mathrm{L}$ of seed is added to the growth solution. The resulting mixture is gently mixed for $15 \mathrm{~s}$ and kept at $28^{\circ} \mathrm{C}$ without disturbance overnight for the growth stage.

The final nanostructure solution is cleaned from excess reactants by two cycles of centrifugation $(8694 \mathrm{~g}, 40 \mathrm{~min}$ ), and sediment is redispersed in pure water. The resulting solution is concentrated by a factor 10 through a final centrifugation to $1 \mathrm{~mL}$.

Different synthetic conditions have been used to promote the anisotropy. First, two different $\mathrm{AgNO}_{3}$ concentrations were used while keeping all the other conditions constant. Second, the $\mathrm{pH}$ of the growth solution was controlled by two different methods: (1) via addition of a small amount $(100 \mu \mathrm{L})$ of $0.1 \mathrm{M} \mathrm{HCl}$ and (2) via the use of 5bromosalicylic acid $(0.01 \mathrm{M})$ with a lower $\mathrm{CTAB}, \mathrm{AA}$, and seed concentration $(0.05 \mathrm{M}, 0.25 \mathrm{mM}$, and $60 \mu \mathrm{L}$, respectively). All the chemical conditions used during preparation of different aspect ratio (AR) bipyramids are presented in the Supporting Information (Table S1 and section 1-ii).

2.2.2. Characterization. 2.2.2.1. UV-vis Spectroscopy. Absorption spectra of final particles were measured in a $1 \mathrm{~mm}$ Helma cell using a Shimadzu UV- 2550 UV-vis spectrophotometer. Data were analyzed with UV Probe Software. 

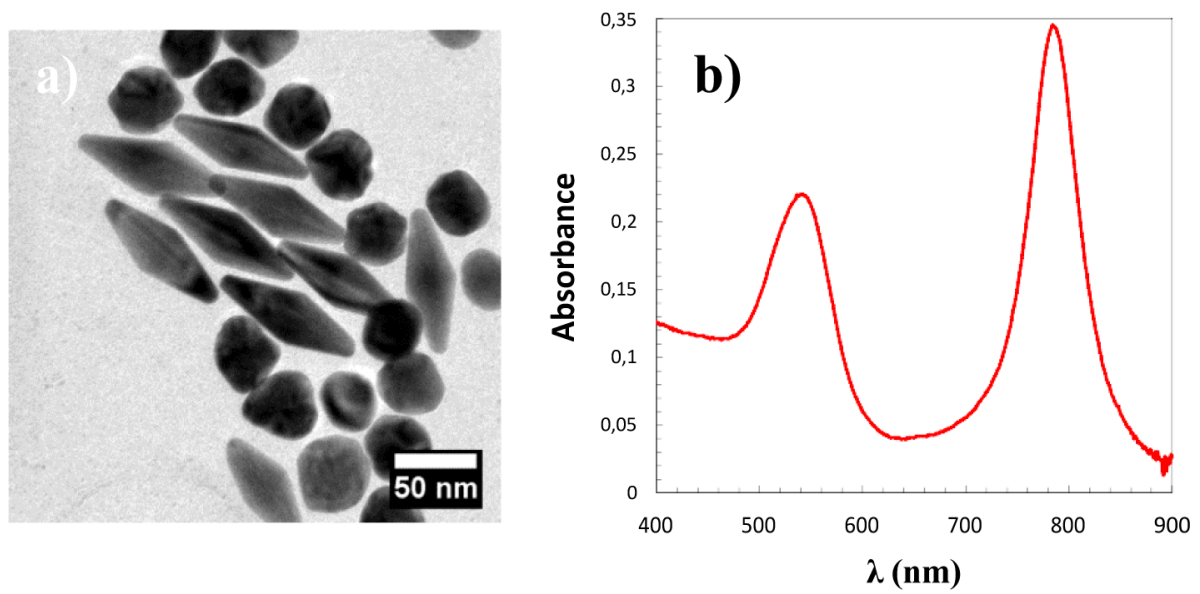

Figure 1. (a) TEM image of Au bipyramids synthesized by addition of $60 \mu \mathrm{L}$ of seed solution into growth solution including 5-bromosalicylic acid (AR $\approx$ 2.7) (Table S1, no. 3 experimental conditions detailed in Supporting Information, section 1-ii) and (b) corresponding UV-vis spectra of the solution.
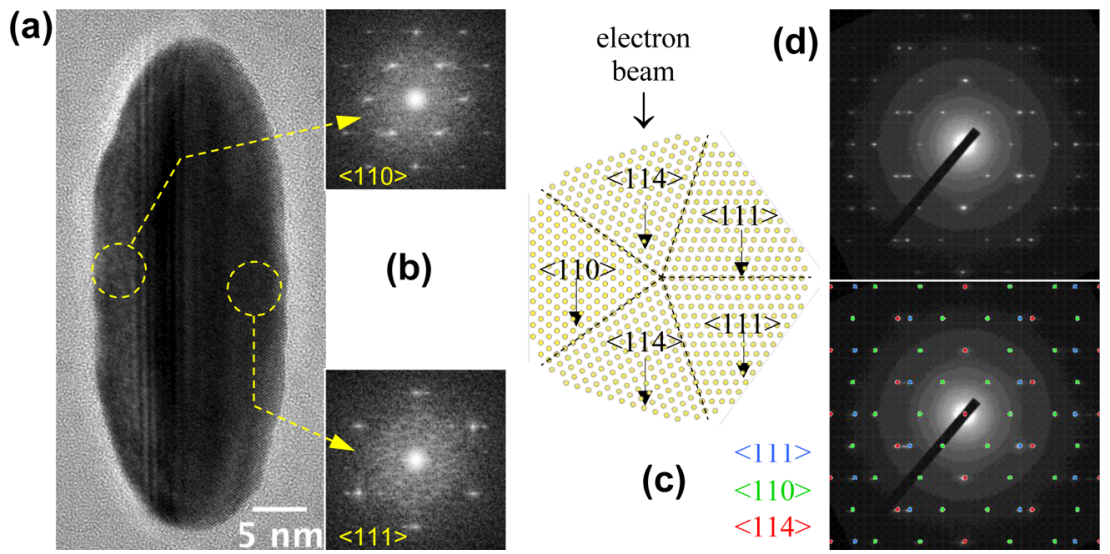

Figure 2. (a) HRTEM observation of a large bipyramid with elongation direction perpendicular to the electron beam. (b) In this orientation, crystals located on the sides of the bipyramid have different orientations: $\langle 110\rangle$ and $\langle 111\rangle$ zone axis deduced from FFT analysis. (c) This result can be interpreted in agreement with a pentatwinned structure of the bipyramid. (d) The corresponding SAED pattern may be analyzed as the superimposition of $\langle 111\rangle,\langle 110\rangle$, and $\langle 114\rangle$ zone axis diffraction pattern.

2.2.2.2. Transmission Electron Microscopy (TEM). Characterization of the nanoparticles was performed by TEM. Low resolution electron observations were carried out using a Philips CM 30 operating at $300 \mathrm{kV}$. An $8 \mu \mathrm{L}$ drop of concentrated gold nanoparticle solution was dried on a carbon coated copper grid overnight before TEM analysis. HRTEM observations were achieved using two different microscopes: a JEOL $2100 \mathrm{~F}$ microscope equipped with Gatan US4000 CCD camera operating at $200 \mathrm{kV}$ (installed at IMPMC, Paris UPMC) and a JEOL ARM $200 \mathrm{~F}$ Cold FEG equipped with a CEOS Cs-image corrector operating at $200 \mathrm{kV}$ and $80 \mathrm{kV}$ (installed at MPQ-Université Paris Diderot, Paris France).

2.2.2.3. Electron Tomography. Tomography data were acquired in a JEOL 2100F transmission electron microscope with a field emission gun, equipped with a spherical aberration (Cs) probe corrector and operating at $200 \mathrm{kV}$ (installed at ICPMS-Strasbourg, France). The acquisition of bright field (BF) and dark field (DF) tilt series was carried out simultaneously in the scanning mode (STEM), by using a circular detector for the BF and a high angle annular detector for the HAADF. A camera length of $10 \mathrm{~cm}$ was chosen for this experiment. It corresponds to inner and outer semiangles of 60 and $160 \mathrm{mrad}$, respectively, for the HAADF detector. A $100 \mu \mathrm{m}$ condenser aperture was employed, allowing one to reach a probe diameter of about $0.12 \mathrm{~nm}$ with a current density of $0.5 \mathrm{pA} \cdot \AA^{-2}$. Under these conditions, the tomography series were acquired using the Digital Micrograph software (tomography plugin), giving access to an automatic increment of the tilt angles and a sharp control of the specimen drift and defocusing. A high tilt specimen holder from Gatan was employed for a tilting range of $-65^{\circ}$ to $65^{\circ}$, with an equal angular step of $2.5^{\circ}$. From analytical relations described by Midgley et al., ${ }^{23}$ the tomogram resolution was estimated at about 0.16 $\mathrm{nm}$ in the direction perpendicular to the electron beam. For the direction of the electron beam, the resolution diminishes with a factor of 1.22 , due to the missing wedge from data acquisition.

Once the series acquisition was completed and given the high contrasts achieved in the HAADF mode, a cross-correlation algorithm was sufficient to properly align the images from the series. The volume reconstruction was carried out by discrete algebraic reconstruction techniques (DART) ${ }^{24}$ using the Tomo J plugin under the Fiji (ImageJ) software. The same software was used for the volume visualization and analysis.

\section{RESULTS}

The as-prepared bipyramids were produced by the classical silver(I)-seeded growth method. ${ }^{25}$ Using the reference conditions given in section 2.2.1, a dispersion of bipyramids in coexistence with spheres (Supporting Information, Supp.InfoF8) was obtained. We retrieve the polymorphism generally obtained in bipyramid or nanograin syntheses based on the seeded growth method, a consequence of their high sensitivity to reshaping. ${ }^{26,27}$ In the chosen synthetic method, the aspect ratios of the bipyramids were improved by (a) increasing the $\mathrm{AgNO}_{3}$ concentration (Supporting Information, Supp.Info-F1), (b) adding 5-bromosalicylic acid which is classically used for 

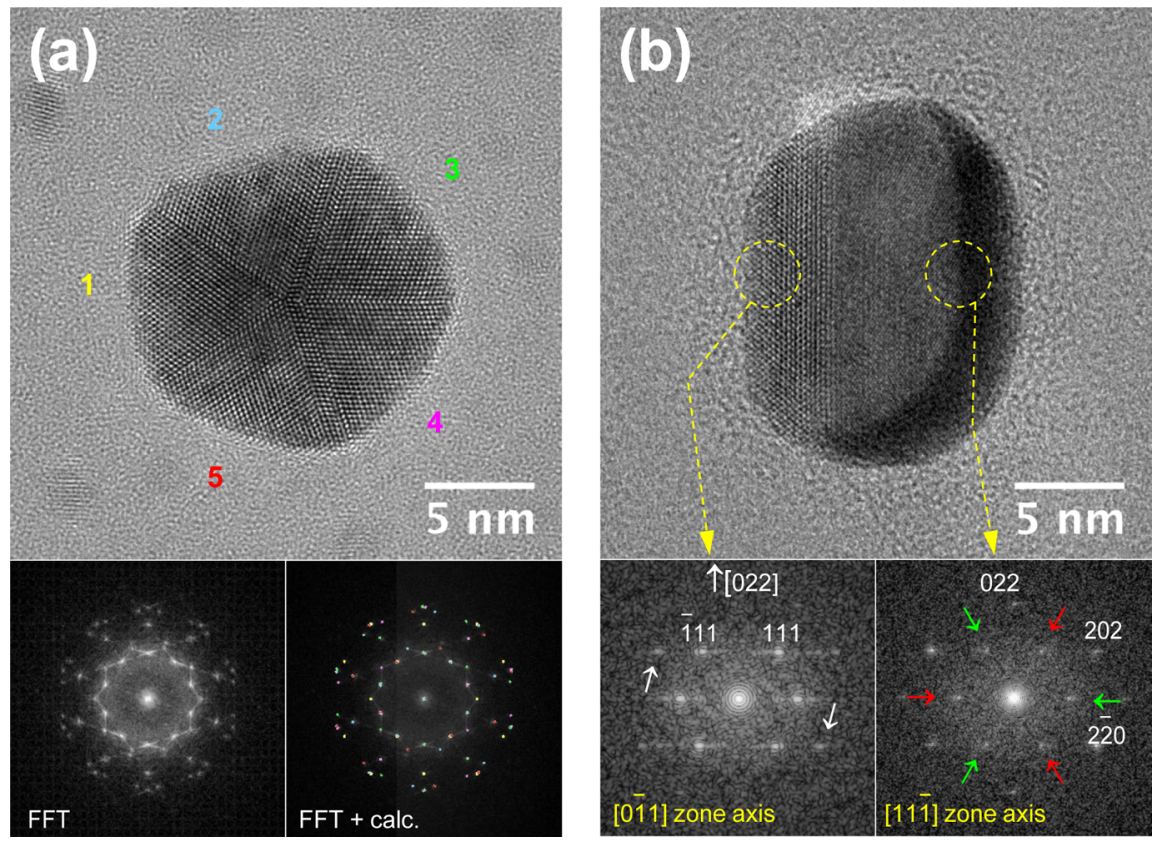

Figure 3. (a) Structural analysis of intermediate sized bipyramids (nanograins) and HRTEM image of the pentatwinned particle, constituting five individual crystals oriented along a common $\langle 110\rangle$ direction (parallel to the electron beam), exhibiting a 5-fold symmetry. The overall fast Fourier transform can be interpreted as the superimposition of five $\langle 110\rangle$ zone axis diffraction patterns (the color of the calculated diffraction pattern is related to the corresponding labeled crystal). (b) HRTEM image of a multitwinned particle constituting at least two crystals oriented along a common $\langle 110\rangle$ direction (perpendicular to the electron beam). The FFT corresponding to the left part of the particle is related to a distorted $\langle 110\rangle$ zone axis diffraction pattern. The white arrows indicate supplementary 220-type reflections likely due to the adjacent grains. The FFT corresponding to the right part of the particle is related to a $\langle 111\rangle$ zone axis diffraction pattern. Red and green arrows indicate supplementary reflections related to upper and lower first order Laue zone for the $[111]$ zone axis.
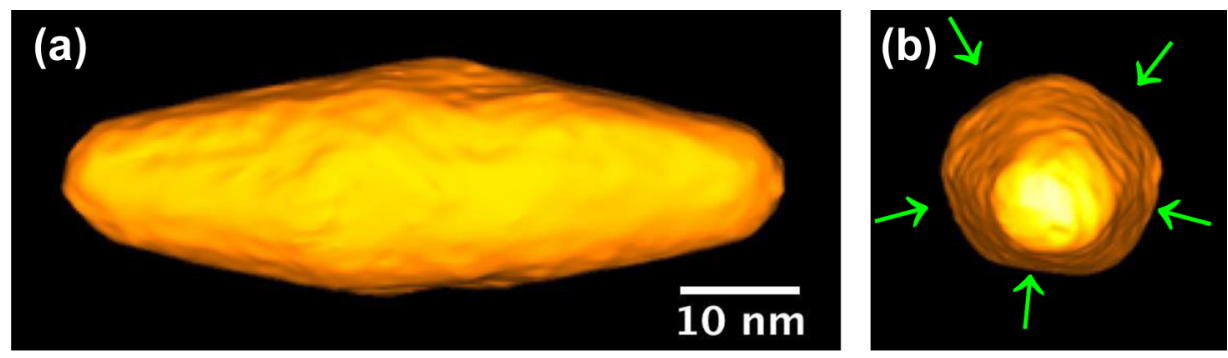

Figure 4. (a) Figure e-tomo 3D volume of a bipyramid obtained from a DART reconstruction procedure and (b) the bipyramid viewed along the elongation direction. A clear pentagonal section is evidenced.

nanorods, ${ }^{28}$ employed here for the first time as an additive to CTAB to produce bipyramids (as shown in Figure 1 with TEM and UV-vis characterizations), (c) decreasing the $\mathrm{pH}^{4,25}$ (Supporting Information, Supp.Info-F2), or (d) decreasing the volume of added seeds ${ }^{4,29}$ (Supporting Information, Supp.InfoF2). In all these conditions, it appears that a rounding of the apexes is generally observed for small aspect ratio bipyramids.

3.1. Morphology of the Bipyramids. The morphology of the bipyramids has been obtained from the analysis of HRTEM images and SAED patterns. Bipyramids with large aspect ratios $(A R \geq 2)$ and sizes greater than $20 \mathrm{~nm}$ are most often observed with the elongation direction parallel to the carbon grid, that is, perpendicular to the electron beam (Figure 2, Supporting Information, Supp.Info-F3 and -F4). They are always characterized by a nonuniform contrast and the presence of planar defects. From the analysis of several nanoparticles, it appears that the large bipyramids are always twinned and only two main observation directions separated by $18^{\circ}$ are accessible (Figure 2; Supporting Information, Supp.Info-F3 and -F4, for other orientations and bipyramids). For the particle shown in Figure $2(D=17.1 \mathrm{~nm}, L=45.7 \mathrm{~nm}, \mathrm{AR}=2.7)$, two crystal orientations of the multitwinned bipyramid can be deduced from the fast Fourier transform (FFT) analysis, $\langle 110\rangle$ and $\langle 111\rangle$ (Figure 2b). The SAED pattern corresponds to a superimposition of three, $\langle 111\rangle,\langle 110\rangle$, and $\langle 114\rangle$, zone axis diffraction patterns, which is consistent with the multiple twinning of a face-centered cubic structure (Figure 2c).

This type of twinning, common in isotropic gold nanoparticles with decahedral (i-Dh) morphologies, ${ }^{30-33}$ has also been observed for gold ${ }^{34}$ and copper ${ }^{5}$ nanorods. We can conclude that the large bipyramids are based on pentatwinned structures showing agreement with the initial TEM observations by Liu and Guyot-Sionnest. $^{12}$

In contrast, smaller bipyramids (size $\leq 20 \mathrm{~nm}$ ) with smaller aspect ratios offer a wider range of orientations. Thus, this type of particle may be observed, as in Figure 3a, with an apparent 5-fold axis almost parallel to the electron beam (i.e., with an angular shift within the tilt capabilities of the double tilt specimen holder) 

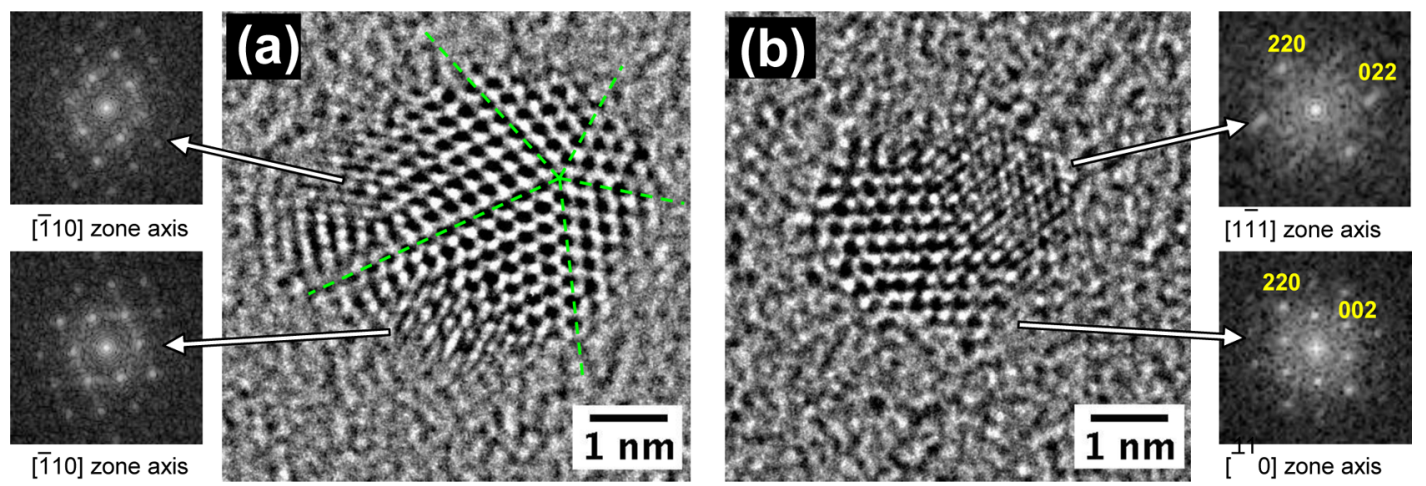

Figure 5. Structural analysis of twinned seeds. (a) Ultra-HRTEM image of a $3.3 \mathrm{~nm}$ particle with FFT analysis of selected areas. (b) Ultra-HRTEM image of a $2.7 \mathrm{~nm}$ seed particle with different orientation.

or as in Figure 3b, exhibiting a similar orientation observed for large sized bipyramids (as in Figure 2). From the FFT analysis, a pentatwinned structure can be unambiguously attributed to low aspect ratio bipyramids (also called "nanograins") $(D=11.9 \mathrm{~nm}$, $L=16.9 \mathrm{~nm}, \mathrm{AR}=1.4$ for nanograin given in Figure $3 \mathrm{~b}$ ). Hence, such typical twinning is observed for different elongated nanoparticles with different aspect ratios going from nanograins (small aspect ratio) to bipyramids (large aspect ratio).

To completely assess the internal structure of the bipyramids described above, a simulated model of a pentatwinned bipyramid of ca. 3000 atoms exhibiting high index $\{11 n\}$ stepped side faces was produced. The different views (Supporting Information, Supp.Info-F6) from varied orientations reproduce the observed TEM images of bipyramids. The variation in the area of the twinning zone along the center of the particles results from the contribution of the different crystals oriented along the horizontal axis (experimental differences are visible from comparison between Figure 2a and Supp.Info-F3a and -F4a, Supporting Information).

Further observations were achieved by STEM-HAADF electron tomography on a large size bipyramid. Figure 4 shows the 3-D morphology reconstructed from DART algorithms. ${ }^{24}$ The bipyramid ( $D=18 \mathrm{~nm}, L=64 \mathrm{~nm}, \mathrm{AR}=3.5)$ presents rounded tips with an angle of $27^{\circ}$ between the side edges. Our results differ significantly from those obtained by Burgin et al. ${ }^{21}$ who observed a hexagonal section. In our case, despite the fact that some angles may be rounded due to the missing wedge, a clear pentagonal section can be extracted.

3.2. Characterization of Seeds and Small Size Nanoparticles. The low concentration of seeds associated with their small size make their shape and structure analysis quite difficult. Contrary to the Turkevich method known to produce stable isolated nanoparticles, ${ }^{35}$ the small nanoparticles produced by borohydride addition are quite unstable. Combinations of DLS, SAXS, and UV-vis spectroscopy (Supporting Information, Supp.Info-F7), used on a freshly prepared solution, evidence a large size distribution $(\sigma / R=0.37)$ of NPs with an average particle size of $5 \mathrm{~nm}$. Further attempts for direct TEM analysis on these NPs lead to the formation of large sized aggregates on the TEM support. To overcome such a problem, we have imaged the residual seeds unaffected by the reduction of additional metal salt in the growth solution (Supporting Information, Supp.InfoF8) ${ }^{36}$ by assuming that these small particles represent the seeds after their addition to CTAB solution.

A HRTEM image of sub-5 $\mathrm{nm}$ pentatwinned particles found in the final solution after growth is shown in Figure 5a. The particle is constituted in such a way that five individual crystals oriented along a common $\langle 110\rangle$ direction (parallel to the electron beam) exhibit 5-fold symmetry. Similarly, a small, multitwinned nanoparticle shown in Figure $5 \mathrm{~b}$ exhibits the same structural feature as the one described for large and small aspect ratio bipyramids, that is, adjacent subunits with the same relative crystallographic orientation (Figures 2 and 3 ).

It is remarkable that for all the different sizes of nanoparticles representative of the particles in formation, HRTEM images evidence pentatwinned structure either for initial isotropic seeds or for elongated shapes (nanograins and bipyramids).

\section{DISCUSSION}

From HRTEM and SAED results, the structure of gold bipyramids obtained by silver(I)-assisted seeded growth processes have been determined. Analyses have been conducted on a final sample containing different sizes of representative growing nanoparticles. A pentatwinned structure was determined for both highly elongated bipyramids with sizes largely greater than $5 \mathrm{~nm}$ and for the smallest apparently isotropic nanoparticles with sizes below $5 \mathrm{~nm}$.

Previously, Zhou et al. ${ }^{18}$ have shown strong evidence of growth of big decahedral seeds $(25-49 \mathrm{~nm})$ into bipyramids with the conservation of 5-fold twinning. However, for smaller size seeds, such direct evidence of conservation of the pentatwinned structure during growth of bipyramids was not obtained due to size limitations.

For considerably small sizes $(<5 \mathrm{~nm})$, the isotropic decahedral (i-Dh) particles are known to be most stable in vacuum and have been largely described in literature. ${ }^{32,37-39}$ However, in solution containing surface ligands, experimental evidence of this structure is more tricky. ${ }^{12,40,41}$ The use of low voltage $(80 \mathrm{kV})$ during HRTEM observations allowed us to explore the decahedral structure of the small size seeds added in silver(I)assisted synthesis of bipyramids, even in the presence of CTAB surfactant. From these experiments, we show the conservation of the pentatwinned structure during the growth of seeds into bipyramids.

It is known that for a face-centered cubic structure, the only way to obtain elongated shapes is to break the symmetry by incorporating twinning defects or stacking faults into the nanocrystal. ${ }^{36,42}$ For nanoparticles in solution, the ligands can promote this process by inverting the energy of different facets through selective adsorption. In the CTAB silver(I) solution, small, elongated nanoparticles bearing pentatwinned structures with extended $\{100\}$ facets are observed in comparison to the initial i-Dh structure of seeds (Figure 5, see Supporting Information Supp.Info-F9 and -F10 for details). 
There have been theoretical attempts to account for the modification of surface energies of certain facets on gold NPs in the presence of surfactants and solution ions. Almora-Barrios et $\mathrm{al}^{43}$ have analyzed the role of silver on gold surface stability, in relation to experimental features. The promotion of the monocrystalline nanorod anisotropy experimentally results from the combination of face-capping by the $\mathrm{CTA}^{+} /\left[\mathrm{AgBr}_{2}\right]^{-}$ ions ${ }^{15,44}$ (hereafter called CTASB) and the stabilization effect of the CTAB/CTASB surfactant double layer. ${ }^{45}$ Using density functional theory simulations, they demonstrate the preferential adsorption of the CTASB complex on open $\{100\}$ facets on a gold nanorod surface in comparison to $\{111\}$ facets, and its strong stabilizing effect for higher index $\{520\}$ facets presented on the lateral sides of the monocrystalline nanorods. Beyond arguments on surface energies by the use of a simplified atomistic model of CTAB, Grochola et al. ${ }^{46}$ have simulated the adsorption of the surfactant on gold nanoparticles to explain the growth of nanorods by preferential adsorption on chosen facets.

At this stage, in order to account for multitwinned nanorod or bipyramid stability, arguments based solely on surface energies (and deduced Wulff shapes) turn out to be insufficient. On the one hand, for small size NPs that are in the first stages of growth, besides surface energies, the energy contributions of edges and corners are important. On the other hand, the presence of twinning planes and of a 5-fold symmetry axis destabilizes the core of the particles as their size increases. This energy term is an important contribution, absent in monocrystalline particles.

Conversely, stability of the multitwinned gold nanorods or gold bipyramids in a complex environment can be qualitatively accounted for by using the atomistic approach for metalenvironment interaction that we have recently developed. ${ }^{47}$ The total energy $(E)$ of a NP is expressed as the sum of many-body gold-gold and gold-environment interactions. The former interaction is given by the second-moment approximation (SMA) to the tight binding Hamiltonian, ${ }^{48}$ and the goldenvironment interaction is the sum of all under-coordinated atoms $i$ of the form: eq 1 .

$$
E_{i}^{\mathrm{M}-\mathrm{E}}=-\epsilon\left(Z_{\mathrm{B}}-Z_{i}\right)^{p}
$$

The difference $Z_{\mathrm{B}}-Z_{i}$ between the coordination number $Z_{\mathrm{B}}$ in the bulk $\left(Z_{\mathrm{B}}=12\right.$ for gold fcc $)$ and the $Z_{i}$ of an atom $i$ is equal to the number of free bonds available to bind to species in the environment. The pair of positive parameters $(\epsilon, p)$ defines the strength and the nature of the gold-environment interaction. $^{47,49}$

In order to focus on gold pentatwinned nanostructures, a motif optimization technique ${ }^{50,51}$ was used to generate families of closed-shell NPs in the size range $2500<N<3500$ atoms, terminated by different proportions of $\{111\},\{100\},\{110\}$, $\{311\}$, and $\{210\}$ facets. For each initial configuration, quenched molecular dynamics runs were performed under conditions that preserve their shape and symmetry. In line with the conclusions of our recent study, ${ }^{49}$ we have found two sets of parameters $(\epsilon, p)$ for which the reduction of NP surface and edge energies due to the interaction with the environment diminishes drastically the stability gap between elongated particles and the isotropic decahedral seeds (i-Dh).

Figure 6 displays the evolution of the excess energy of the most stable particles with respect to an equal number $N$ of bulk atoms (energy $E_{\text {coh }}$ ) defined as

$$
\Delta(N)=\frac{E_{\mathrm{NP}}(N)-N E_{\mathrm{coh}}}{N^{2 / 3}}
$$

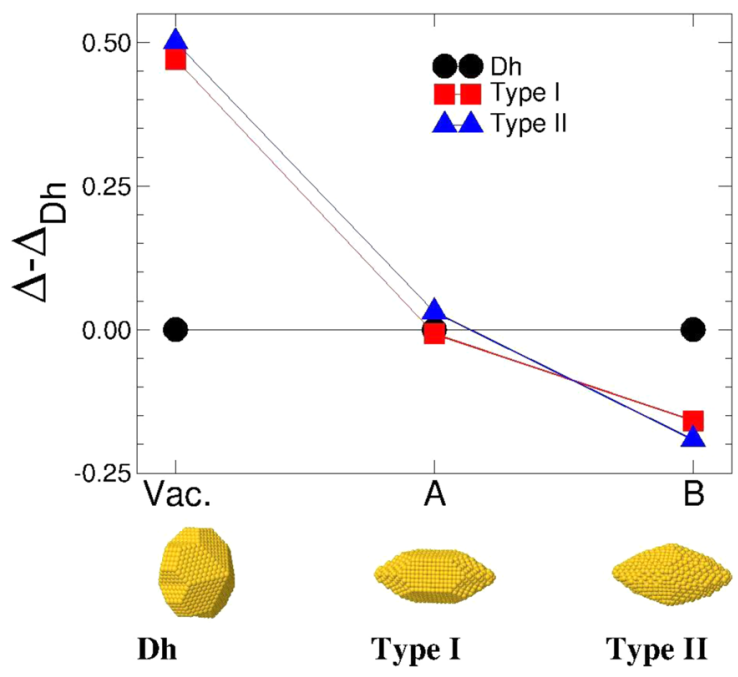

Figure 6. (top) Excess surface energies of the most stable elongated pentatwinned gold particles with respect to the isotropic decahedral (i$\mathrm{Dh}$ ) ones $\Delta-\Delta_{\mathrm{Dh}}\left(\mathrm{eV} / \mathrm{N}^{2 / 3}\right)$. (bottom) i-Dh, type I, and type II particles corresponding, respectively, to the most stable structures in vacuum and environments $A$ and $B$, created with an average of 3000 gold atoms per particle.

in vacuum and in environments $\mathrm{A}(\epsilon=0.05 \mathrm{eV} /$ atom, $p=1.5)$ and $\mathrm{B}(\epsilon=0.022 \mathrm{eV} /$ atom, $p=2.0)$, as well as their equilibrium shapes. Environments A and B favor $\{100\}$ and high order $\{210\}$ facets, respectively, which qualitatively mimics ab initio results of Almora-Barrios et $\mathrm{al}^{43}$ for CTAB and CTASB adsorption on gold, respectively. Even though the strength, $\epsilon$, of the goldenvironment interaction is smaller in environment $\mathrm{B}$, it is more strongly coordination dependent.

In that way, the weight of the edges over facets is higher in environment $\mathrm{B}$ than in $\mathrm{A}$.

In environment $A$, the NP has an aspect ratio of 2.2 , with fairly long lateral $\{100\}$ facets and relatively small tips bordered by five $\{110\}$ facets. Their shape is that of pentatwinned nanorods, similar to those observed by Johnson and co-workers, ${ }^{34}$ with largely extended $\{100\}$ side facets. The pentatwinned nanorods bearing large $\{100\}$ facets can experimentally be obtained in a very low yield $(<1 \%)$ by the growth of citrate seeds in the absence of silver nitrate. ${ }^{8,25}$ Such low yield can be explained by the fact that only a few seeds developing small $\{100\}$ facets in relatively small sizes can be transformed into the small nanorods. As soon as the anisotropy is developed, gold nanorods continue to grow by increasing their $\{100\}$ surfaces of the lateral facets by an autocatalytic process. ${ }^{34}$ In this case, higher index facets are not stabilized and the transition of small nanorods into bipyramids cannot be observed.

In environment B, particles with an aspect ratio of 1.9 are stabilized. At their tips, these NPs expose $\{210\}$ facets, representative of higher index facets. These pentatwinned NPs resemble the so-called nanograins (low AR bipyramids, shown in Figure 3 and Supporting Information Supp.Info-F5) obtained in experiments based on silver-(I)-assisted growth of citrate seeds (i.e., when CTASB $\left(\mathrm{CTA}^{+} /\left[\mathrm{AgBr}_{2}\right]^{-}\right)$is present in the growth solution). In this case, higher facets are stabilized, and bipyramid shapes are favored. We have seen experimentally that nanograins and bipyramids bear the same internal structure (Figures 2 and 3 ). We can thus assume that as soon as nanograins are formed, further monomer feeding can proceed along twinning axis along the tip of the nanoparticles (due to less compact surfactant 


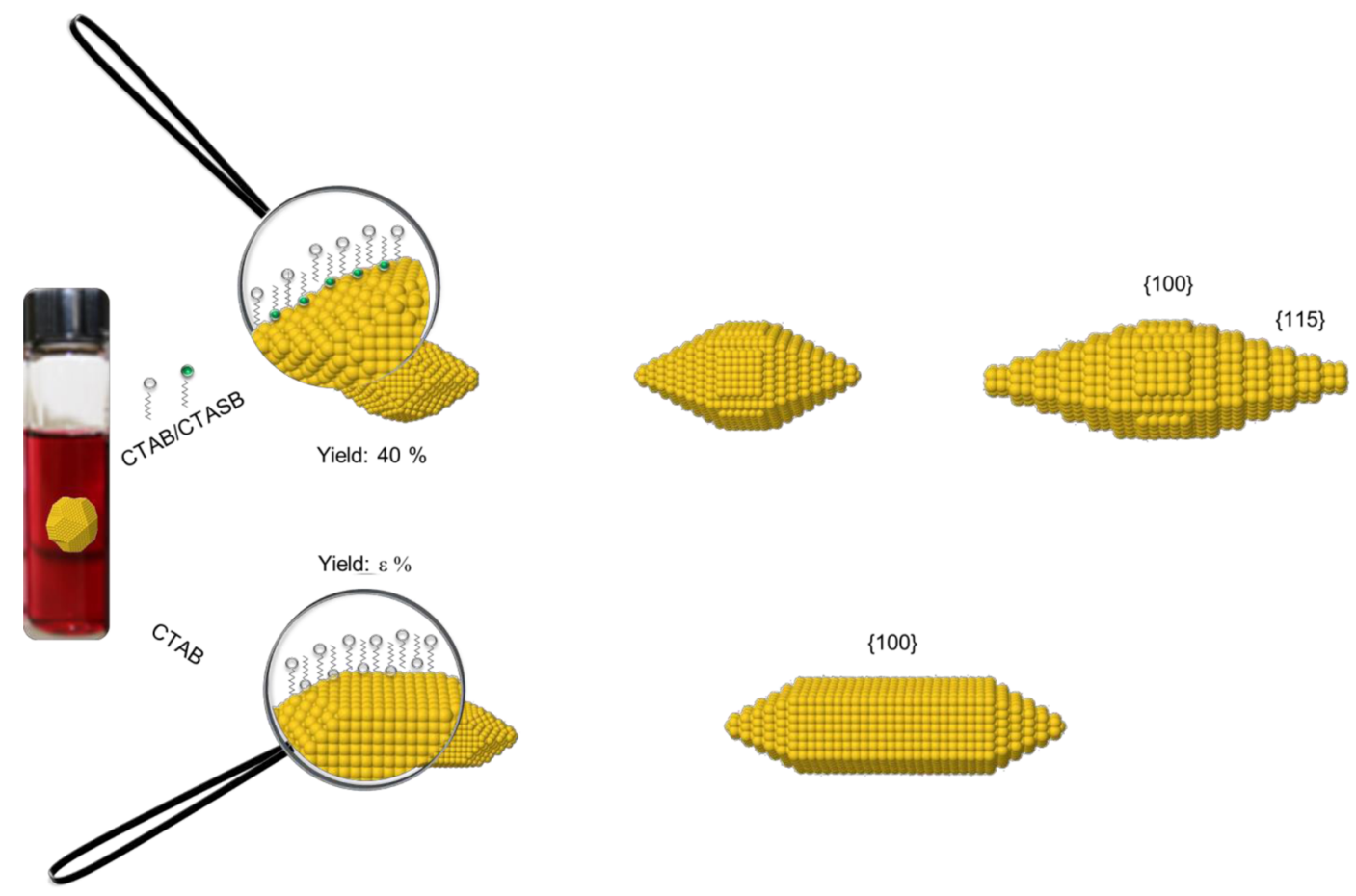

Figure 7. Formation mechanism of bipyramids and nanorods. In the present case, the bipyramids are formed by a silver(I)-assisted process in CTAB solution and deduced from HRTEM; pentatwinned structured is maintained along the growth. For pentatwinned nanorod formation, they are obtained without silver from decahedra seeds in a very low yield.

layers ${ }^{29}$ ) leading to an increase in the surface of high index facets (which can be stabilized by the CTASB). The nanograins then can continue to evolve into higher aspect ratio bipyramids.

The two growth mechanisms are summarized in Figure 7, highlighting the growth of decahedral isotropic seeds into either gold pentatwinned nanorods without silver in the CTAB growth solution or bipyramids when silver is present. This schematic representation illustrates the inherited structure of the final nanoparticles from initial seeds as a function of the added ligand to the growth solution (i.e. high index versus low index facet stabilization by external ligands).

\section{CONCLUSION}

From a synthetic aspect, we have demonstrated that 5bromosalicylic acid, which is known to promote anisotropy in gold nanorod synthesis, can also be used as an additive in a CTAB environment to promote anisotropy of bipyramids. From a structural aspect, by HRTEM and electron tomography, the internal structure of the bipyramids was determined and definitively attributed to a pentatwinned structure. Even though this structure was initially proposed in the literature, ${ }^{12}$ recent tomographic studies have opened the way to discuss another possible hexagonal symmetry for the bipyramid structure. ${ }^{21}$ Here, we have ruled out this option for the gold bipyramids synthesized in CTAB solution from a silver(I)-assisted growth process. Through a complete HRTEM, SAED, and tomography study, we have also investigated different size NPs from initial decahedral seeds to the final bipyramidal shape. The pentatwinned structure was demonstrated for all these intermediate nanoparticles. Our experimental approach, combined with an atomistic model and taking into account the environment of the gold nanoparticle, has indeed shown that different kinds of elongated nanoparticles (either nanorods or bipyramids) can be stabilized by changing the nature of the ligand used during the synthesis. This consolidates the importance of a fine-tuning of surface chemistry for the control of (exotic) shaped nanoparticles.

\section{ASSOCIATED CONTENT}

\section{Supporting Information}

Further chemical conditions used to synthesize varied aspect ratio bipyramids with additional diffraction analysis and a summary of the evolution of structure is provided. The Supporting Information is available free of charge on the ACS Publications website at DOI: 10.1021/acs.cgd.5b00121.

\section{AUTHOR INFORMATION Corresponding Author \\ *E-mail: fabienne.testard@cea.fr.}

\section{Notes}

The authors declare no competing financial interest.

\section{ACKNOWLEDGMENTS}

The authors thank Olivier Taché for the 3D printed nanoparticles used in the graphical abstract. The authors acknowledge the French CNRS (Centre National de la Recherche Scinetifique) (Grant FR3507) and CEA (Commissariat à l'Energie Atomique et aux Energies Alternatives) METSA (Microscopie Electronique et Sonde Atomique) network. The authors acknowledge the financial support from Agence Nationale de la Recherche (ANR) under Contract ANR11 BS10 006. This work has been supported by the Region Ile-de-France in the framework of DIM C'Nano IdF.

\section{REFERENCES}

(1) Gole, A.; Murphy, C. J. Chem. Mater. 2004, 16, 3633-3640.

(2) Pérez-Juste, J.; Pastoriza-Santos, I.; Liz-Marzán, L. M.; Mulvaney, P. Coord. Chem. Rev. 2005, 249, 1870-1901. 
(3) Chen, H.; Gao, Y.; Zhang, H.; Liu, L.; Yu, H.; Tian, H.; Xie, S.; Li, J. J. Phys. Chem. B 2004, 108, 12038-12043.

(4) Grzelczak, M.; Perez-Juste, J.; Mulvaney, P.; Liz-Marzan, L. M. Chem. Soc. Rev. 2008, 37, 1783-1791.

(5) Lisiecki, I.; Filankembo, A.; et al. Phys. Rev. B: Condens. Matter Mater. Phys. 2000, 61, 4968-4974.

(6) Huang, X.; El-Sayed, M. A. J. Adv. Res. 2010, 1, 13-28.

(7) Jana, N. R.; Gearheart, L.; Murphy, C. J. Adv. Mater. 2001, 13, 1389-1393.

(8) Wang, Z. L.; Mohamed, M. B.; Link, S.; El-Sayed, M. A. Surf. Sci. 1999, 440, L809-L814.

(9) Sau, T. K.; Murphy, C. J. J. Am. Chem. Soc. 2004, 126, 8648-8649.

(10) Kawamura, G.; Nogami, M. J. Cryst. Growth 2009, 311, 44624466

(11) Liu, M.; Guyot-Sionnest, P.; Lee, T.-W.; Gray, S. K. Phys. Rev. B: Condens. Matter Mater. Phys. 2007, 76, 235428.

(12) Liu, M.; Guyot-Sionnest, P. J. Phys. Chem. B 2005, 109, 2219222200 .

(13) Johnson, C. L.; Snoeck, E.; Ezcurdia, M.; Rodriguez-Gonzalez, B.; Pastoriza-Santos, I.; Liz-Marzan, L. M.; Hytch, M. J. Nat. Mater. 2008, 7, $120-124$.

(14) Jana, N. R. Small 2005, 1, 875-882.

(15) Hubert, F.; Testard, F.; Spalla, O. Langmuir 2008, 24, 9219-9222.

(16) Carbó-Argibay, E.; Rodríguez-González, B.; Gómez-Graña, S.; Guerrero-Martínez, A.; Pastoriza-Santos, I.; Pérez-Juste, J.; Liz-Marzán, L. M. Angew. Chem., Int. Ed. 2010, 49, 9397-9400.

(17) Katz-Boon, H.; Rossouw, C. J.; Weyland, M.; Funston, A. M.; Mulvaney, P.; Etheridge, J. Nano Lett. 2011, 11, 273-278.

(18) Zhou, G.; Yang, Y.; Han, S.; Chen, W.; Fu, Y.; Zou, C.; Zhang, L.; Huang, S. ACS Appl. Mater. Interfaces 2013, 5, 13340-13352.

(19) Kou, X.; Zhang, S.; Tsung, C.-K.; Yeung, M. H.; Shi, Q.; Stucky, G. D.; Sun, L.; Wang, J.; Yan, C. J. Phys. Chem. B 2006, 110, 16377-16383.

(20) Kou, X.; Ni, W.; Tsung, C.-K.; Chan, K.; Lin, H.-Q.; Stucky, G. D.; Wang, J. Small 2007, 3, 2103-2113.

(21) Burgin, J.; Florea, I.; Majimel, J.; Dobri, A.; Ersen, O.; TreguerDelapierre, M. Nanoscale 2012, 4, 1299-1303.

(22) Yoo, H.; Jang, M. H. Nanoscale 2013, 5, 6708.

(23) Midgley, P. A.; Weyland, M. Ultramicroscopy 2003, 96, 413-431.

(24) Herman, G. T.; Kuba, A. Discrete Tomography: Foundations, Algorithms, and Applications Series: Applied and Numerical Harmonic Analysis; Birkhäuser: Basel, 1999; Vol. XXII.

(25) Jana, N. R.; Gearheart, L.; Murphy, C. J. J. Phys. Chem. B 2001, 105, 4065-4067.

(26) Jana, N. R.; Gearheart, L.; Obare, S. O.; Murphy, C. J. Langmuir 2002, 18, 922-927.

(27) Zheng, Y.; Zeng, J.; Ruditskiy, A.; Liu, M.; Xia, Y. Chem. Mater. 2014, 26, 22-33.

(28) Ye, X.; Jin, L.; Caglayan, H.; Chen, J.; Xing, G.; Zheng, C.; DoanNguyen, V.; Kang, Y.; Engheta, N.; Kagan, C. R.; Murray, C. B. ACS Nano 2012, 6, 2804-2817.

(29) Lohse, S. E.; Murphy, C. J. Chem. Mater. 2013, 25, 1250-1261.

(30) Iijima, S.; Ichihashi, T. Phys. Rev. Lett. 1986, 56, 616-619.

(31) Koga, K.; Sugawara, K. Surf. Sci. 2003, 529, 23-35.

(32) Li, Z. Y.; Young, N. P.; Di Vece, M.; Palomba, S.; Palmer, R. E.; Bleloch, A. L.; Curley, B. C.; Johnston, R. L.; Jiang, J.; Yuan, J. Nature 2008, 451, 46-48.

(33) Marks, L. D. Rep. Prog. Phys. 1994, 57, 603.

(34) Johnson, C. J.; Dujardin, E.; Davis, S. A.; Murphy, C. J.; Mann, S. J. Mater. Chem. 2002, 12, 1765-1770.

(35) Enustun, B. V.; Turkevich, J. J. Am. Chem. Soc. 1963, 85, 33173328 .

(36) Lofton, C.; Sigmund, W. Adv. Funct. Mater. 2005, 15, 1197-1208.

(37) Hofmeister, H. Z. Kristallogr. 2009, 224, 528-538.

(38) Reyes-Gasga, J.; Elechiguerra, J. L.; Liu, C.; Camacho-Bragado, A.; Montejano-Carrizales, J. M.; Jose Yacaman, M. J. Cryst. Growth 2006, $286,162-172$.

(39) Howie, A.; Marks, L. D. Philos. Mag. A 1984, 49, 95-109.

(40) Xu, X. J.; Saghi, Z.; Inkson, B. J.; Möbus, G. J. Nanopart. Res. 2010, $12,1045-1053$.
(41) Elechiguerra, J. L.; Reyes-Gasga, J.; Yacaman, M. J. J. Mater. Chem. 2006, 16, 3906-3919.

(42) Xia, Y.; Xiong, Y.; Lim, B.; Skrabalak, S. E. Angew. Chem., Int. Ed. 2009, 48, 60-103.

(43) Almora-Barrios, N.; Novell-Leruth, G.; Whiting, P.; Liz-Marzán, L. M.; López, N. Nano Lett. 2014, 14, 871-875.

(44) Niidome, Y.; Nakamura, Y.; Honda, K.; Akiyama, Y.; Nishioka, K.; Kawasaki, H.; Nakashima, N. Chem. Commun. 2009, 13, 1754-1756.

(45) Gómez-Graña, S.; Hubert, F.; Testard, F.; Guerrero-Martínez, A.; Grillo, I.; Liz-Marzán, L. M.; Spalla, O. Langmuir 2012, 28, 1453-1459.

(46) Grochola, G.; Russo, S. P.; Snook, I. K. J. Chem. Phys. 2007, 126, 164707.

(47) Cortes-Huerto, R.; Goniakowski, J.; Noguera, C. J. Chem. Phys. 2013, 138, 244706.

(48) Pettifor, D. G. Bonding and Structure of Molecules and Solids; Clarendon Press: Oxford, U.K., 1995.

(49) Cortes-Huerto, R.; Goniakowski, J.; Noguera, C. Phys. Chem. Chem. Phys. 2015, 17, 6305-6313.

(50) Baletto, F.; Ferrando, R.; Fortunelli, A.; Montalenti, F.; Mottet, C. J. Chem. Phys. 2002, 116, 3856-3863.

(51) Vervisch, W.; Mottet, C.; Goniakowski, J. Phys. Rev. B: Condens. Matter Mater. Phys. 2002, 65, 245411. 